\title{
Decision making in venous thromboembolism prophylaxis: Is LWMH being inappropriately withheld from patients admitted with chronic liver disease?
}

\author{
Authors: Clement Lau, ${ }^{A}$ Christian Burd, ${ }^{B}$ Daniel Abeles $^{\mathrm{C}}$ and David Sherman ${ }^{\mathrm{D}}$
}

\begin{abstract}
Although chronic liver disease (CLD) constitutes a significant proportion of acute medical admissions, it is not known how CLD influences venous thromboembolism (VTE) prophylaxis decision making and low molecular weight heparin (LMWH) prescription. Furthermore, recent evidence suggests that VTE risk has been underestimated in CLD and that prophylactic LMWH is safe and may improve outcome in this patient group. We therefore evaluated VTE prophylaxis in patients with CLD and aimed to determine the factors contributing to decisions to prescribe LMWH. Prescription of LMWH was significantly less likely in CLD patients than in general medical patients ( $29 \%$ vs $55 \%$; $p<0.01$ ). Patients with CLD who were prescribed LMWH were more likely to have been admitted for a 'non-liver' reason than those that did not receive $\mathrm{LMWH}(19 \%$ vs $52 \%$; $\mathrm{p}<0.01)$. These preliminary results show that many patients admitted with CLD, who may benefit from LMWH prophylaxis, do not receive this therapy, because of perceived contraindications for which there may be little evidence. Decision making appears to be affected by whether an admission is 'liver' or 'non-liver' related. Prophylactic LMWH was safe in this small cohort. Further studies are warranted to further inform LMWH prescription in CLD.
\end{abstract}

KEYWORDS: VTE prophylaxis, chronic liver disease, cirrhosis, coagulopathy

\section{Introduction}

The obligatory assessment of venous thromboembolism (VTE) risk on admission and use of appropriate prophylactic measures, including prescription of low molecular weight

\footnotetext{
Authors: ${ }^{\mathrm{A} C T} 2$ doctor, Gastroenterology and GI Surgery Centre, Central Middlesex Hospital, London, UK; ${ }^{\mathrm{B} F Y 1}$ doctor, Gastroenterology and GI Surgery Centre, Central Middlesex Hospital, London, UK; ' specialist registrar in gastroenterology, Gastroenterology and GI Surgery Centre, Central Middlesex Hospital, London, UK; ${ }^{D}$ consultant physician, gastroenterologist and hepatologist, Gastroenterology and GI Surgery Centre, Central Middlesex Hospital, London, UK
}

heparin (LMWH), has been standard practice in UK hospitals since 2008. ${ }^{1}$ However, there is no information on how current decision making on VTE prophylaxis is influenced by the presence of chronic liver disease (CLD) and whether the prescription of LMWH is affected.

CLD is frequently complicated by an increased risk of bleeding with thrombocytopaenia and decreased procoagulant factors, reflected in an increased prothrombin time. However, emerging evidence from recent studies show that individuals with CLD are not protected from, and may be at a greater risk of, thrombotic events, including both portal (PVT) and peripheral venous thromboses. ${ }^{2,3}$ Recently Villa et al demonstrated that prophylactic-dose enoxaparin was able to prevent PVT and even improve survival in this patient group. ${ }^{4}$

This paradox may be due to an imbalance in the 'haemostatic equilibrium' between pro- and anti-coagulant factors found in CLD patients. An increased risk of thrombosis may be explained, in part, by a decrease in plasminogen, proteins $\mathrm{C}$ and $\mathrm{S}$, and antithrombin III levels as well as increases in both factor VIII and von Willebrand factor. ${ }^{2,3}$ These alterations offset the deranged function and reduced circulating quantity of platelets, the reduction in factors II, V, VII, IX, X and XI and elevated tissue plasminogen activator levels, all of which combine to increase bleeding tendency. ${ }^{2}$

A number of retrospective studies have shown the incidence of VTE ranges between 0.5 and $6.3 \%$, with approximately double the relative risk of thrombotic events in patients with cirrhosis versus non-cirrhotics. ${ }^{5,6}$ Conventional practice dictates that CLD patients have suboptimal uptake of VTE prophylaxis, due to the perceived risk of bleeding, particularly from the upper gastrointestinal (GI) tract. However, the risk of VTE can often be exacerbated by other factors, such as increasing age, other co-morbidities and reasons for admission.

The aim of this study was to evaluate the practice of VTE prophylaxis in patients with CLD admitted to a district hospital, to determine the factors that may have contributed to decision making in the prescription or withholding of prophylactic LMWH.

\section{Methods}

We carried out a retrospective case note analysis of consecutive admissions of CLD patients to a district general hospital 
between January and December 2012. Pharmacological prophylaxis with LMWH consisted of dalteparin, usually at 5,000 U once daily, unless there was renal impairment or contraindication. Standard trust VTE assessment forms, drug chart prescription forms and laboratory data were collected. Data on severity of liver disease (Child-Pugh and model for end-stage liver disease (MELD)), presence of oesophageal varices and upper GI bleeding were also recorded.

Results were compared with data collected for a concurrent snapshot audit of VTE prophylaxis in 351 consecutive medical patients admitted to the same hospital trust, a cohort which did not include the CLD admissions.

The admission documentation consisted of a standard decision-making proforma, including risk factors for and against VTE prophylaxis. Completeness of prophylaxis documentation was assessed and categorised into full, partial or absent: full was defined by completion of the risk assessment and a documented decision by the admitting clinician; partial or absent documentation was defined as only partial or no documentation of the risk assessment and/or decision reached respectively. Trust standards required completion of the VTE prophylaxis proforma and prescription documented within 24 hours of admission.

Comparison between groups of continuous variables was performed by the Mann-Whitney U test. Comparisons of categorical variables were performed using the $\chi^{2}$ test or Fisher's exact test. Statistical analysis was performed using IBM $^{\circledast}$ SPSS $^{\circledast}$ v20 (IBM; Armonk, NY, USA) and Prism 4 for Macintosh (GraphPad Prism Sotware inc, San Diego, CA, USA). $\mathrm{p}<0.05$ was considered to indicate a statistically significant difference.

\section{Results}

Eighty consecutive admissions with CLD were identified between January and December 2012. The mean age was 59.6 years with 54 males (68\%). Alcoholic liver disease (ALD) was the aetiology in the majority of patients $(62 / 80 ; 78 \%)$. Other aetiologies included viral infection $(1 / 80 ; 1 \%)$, ALD and hepatitis C (1/80; $1 \%)$, cryptogenic causes $(2 / 80 ; 3 \%)$, non-alcoholic steatohepatitis $(6 / 80 ; 7 \%)$ and autoimmune disease $(8 / 80 ; 10 \%)$. The median MELD score was 13 and $89 \%$ of patients were Child-Pugh B or C. Laboratory tests showed a median (interquartile range (IQR)) creatinine level of $84 \mu \mathrm{mol} / \mathrm{l}(63-122)$, haemoglobin level of 108 g/l (89-119), platelet count of $152 \times 10^{9} / 1$ (91-223) and international normalised ratio (INR) of 1.4 (1.1-1.7).

Reasons for admission included ascites in 35\% (28), 'other liver'-related presentations 36\% (29) and 'non-liver' presentations 29\% (23) (Table 1). 'Other liver'-related admissions were alcohol-related presentations, including hepatitis, intoxication or withdrawal (11), elective admissions for gastroscopy surveillance of varices (8), upper GI bleed presenting as haematemesis or melaena (5), hepatic encephalopathy (4) and spontaneous bacterial peritonitis (1). Non-liver presentations were diagnoses unrelated to CLD including chest infection (7), urinary tract infection (5), falls (4), decompensated heart failure (1), costochondritis (1), anaemia (2), refeeding syndrome (1) and uncertain diagnosis (2).

Upper GI endoscopy results were available in 60 (75\%) admissions, of which 33 (55\%) were confirmed to have oesophageal varices.
Table 1. Admission presentation and frequency of

LMWH prescription in CLD patients.

\begin{tabular}{lll} 
Liver-related presentation & $\begin{array}{l}\text { Number of } \\
\text { patients }\end{array}$ & $\begin{array}{l}\text { LMWH } \\
\text { prescribed }\end{array}$ \\
Ascites & 28 & 6 \\
Alcohol hepatitis, intoxication or & 11 & 2 \\
withdrawal & & \\
Elective gastroscopy for variceal & 8 & 1 \\
surveillance & & \\
Upper GI bleed & 5 & 0 \\
Hepatic encephalopathy & 4 & 2 \\
Spontaneous bacterial peritonitis & 1 & 0 \\
$\quad$ Total & 57 & 11 \\
Non-liver presentation & & \\
Chest infection & 7 & 2 \\
Urinary tract infection & 5 & 5 \\
Falls & 4 & 3 \\
Decompensated heart failure & 1 & 1 \\
Costochondritis & 1 & 0 \\
Anaemia & 2 & 0 \\
Refeeding syndrome & 1 & 0 \\
Diagnosis uncertain & 2 & 12 \\
Total & 23 & 23 \\
Overall total & 80 & \\
\hline CD & & 0 \\
\hline
\end{tabular}

$\mathrm{CLD}=$ chronic liver disease; GI, gastrointestinal; $\mathrm{LMWH}=$ low molecular weight heparin

\section{Documentation of VTE prophylaxis}

According to current national criteria, liver disease is neither a contraindication to nor an indication for pharmacological VTE prophylaxis. ${ }^{7}$ The admitting clinician is required to make a decision whether or not to prescribe LMWH based on standard risk factors.

Documentation of indications for VTE prophylaxis was full, partial or absent in $56 \%, 36 \%$ and $9 \%$ of cases, respectively. Contraindications to $\mathrm{LMWH}$ were documented as: 'no contraindication', 19\%; 'liver disease', $28 \%$; 'other contraindication', $16 \%$; unrecorded, $37 \%$.

\section{Prescribing practice}

Prescription of LMWH was significantly less likely in CLD patients compared with medical patients (23/80 vs 194/351; $29 \%$ vs $55 \%$; $<<0.01$; Fig 1 ). Patients with CLD who were prescribed LMWH were more likely to have been admitted for a 'non-liver' reason compared with those that did not receive $\mathrm{LMWH}(11 / 57$ vs $12 / 23$; 19 vs $52 \%$; $\mathrm{p}<0.01$; Fig 1$)$. Importantly, there were no differences in liver disease severity scores between liver and non-liver groups (median MELD score, 13 vs 12; Child-Pugh C score, 40/57 (70\%) vs 17/23 $(74 \%))$. 


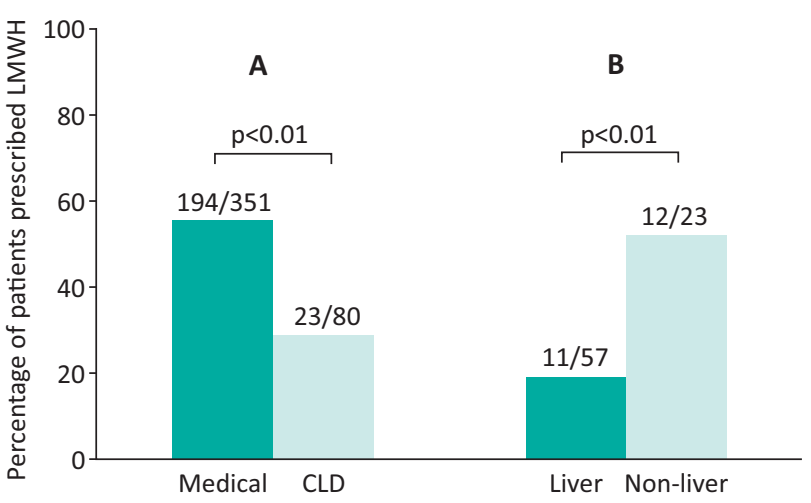

Fig 1. Comparison of LMWH prescription in medical versus CLD patients and in CLD patients presenting with a liver or non-liverrelated condition. (A) Medical vs CLD patients; (B) CLD patients presenting with a liver or non-liver related condition. $C L D=$ chronic liver disease; $\mathrm{LMWH}=$ low molecular weight heparin.

\section{Prevalence of GI bleeding}

Eight CLD patients (10\%) suffered gastrointestinal bleeding during admission presenting as hematemesis or melaena. Patients receiving LMWH had fewer patients with varices (5/23 $(22 \%)$ vs $27 / 57(47 \%))$ or inpatient gastrointestinal bleeding $(1 / 23(4 \%)$ vs $7 / 57(12 \%))$ than those not receiving it, but these differences did not reach statistical significance.

\section{Discussion}

This study demonstrates that both assessment and prescribing in CLD patients is suboptimal compared with general medical patients, and that among CLD patients, those presenting with a 'non-liver'-related condition were more likely to receive VTE prophylaxis than those presenting with a 'liver'-related condition. Our findings support the suggestion that the perceived increase in bleeding risk in all CLD patients affects the assessment of VTE risk and results in LMWH being withheld in a significant proportion. Recent studies also demonstrate low rates of LMWH prescription ranging between 9 and $15 \% .{ }^{8,9}$

The safety of LMWH in patients with CLD has not been validated and there are no randomised controlled trials in the present literature. In a similar retrospective case series analysis, Intagliata et al showed that rates of GI bleeding and death were not increased with the use of pharmacological prophylaxis in a cohort of CLD patients with a mean admission MELD of 16, an INR of 1.46, and normal platelet counts and renal function. ${ }^{10}$ This is similar to our findings, with no significant increase in bleeding prevalence in CLD patients receiving LMWH. The INR threshold below which it would be safe to prescribe prophylactic LMWH still remains to be determined. However, several studies have found no correlation between increasing INR and protection against VTE. ${ }^{7}$

Limitations of this study include the incomplete nature of the documentation referred to in the results section, reflecting the 'real world' nature of the study, which was also a feature of the group used for comparison. In addition, LMWH may have been prescribed more than 24 hours after admission, which was not accounted for, and differences in the grades of junior doctor who usually made decisions on prescribing LMWH are likely. As there are no current guidelines on VTE prophylaxis in CLD patients, prescribing behaviour may have been influenced by factors such as local expert opinion, routine practice and whether patients had refused prophylaxis. There is evidence that prophylactic VTE prescribing can be affected by various human and systemic factors, such as the misperception of VTE prophylaxis underutilisation and uncertainty about who is responsible for ensuring VTE prophylaxis is prescribed. ${ }^{11}$ In one audit, LMWH prescription delays were partly due to the admitting doctor waiting to discuss the decision with a more senior colleague before prescribing. ${ }^{12}$ In our study, VTE prophylaxis prescribing rates were low in both medical and CLD groups (Fig 1). An assessment of local factors affecting prescribing practice with multiple targeted interventions may be able to improve guideline compliance, such as a multidisciplinary educational approach and pharmacists reminding physicians. ${ }^{11}$ A computer-based clinical decision support system providing recommendations to prescribe VTE prophylaxis has shown to alter physician behaviour and improve prescribing practice. ${ }^{13}$ This may benefit all medical patients, particularly those with CLD in which the VTE risk may be in a grey area and more uncertain.

Although the CLD and general medical groups that we compared were admitted to the same hospital group, they were not part of the same cohort and a proportion of the latter group contained a proportion of CLD patients who were not analysed in detail. Furthermore, patients with 'liver'-related conditions may have had LMWH prophylaxis withheld for reasons other than prescribing behaviour alone, for which this study does not account, as reasons were not recorded. Possible explanations may include the withholding of LMWH for clinical procedures such as abdominal paracentesis, given that a significant proportion presented with ascites. However, if indicated, LWMH would normally have been commenced once clinical procedures had been completed.

Decision-making appears to be affected by whether an admission is 'liver' or 'non-liver' related. Further studies are warranted to further inform LMWH prescription and determine the best approach to VTE prophylaxis in this complex patient group. This study adds to the relatively recent consensus in the literature showing that VTE prophylaxis appears to be generally safe and it should be considered and prescribed when clinically indicated, particularly in those patients with small or no varices and no clinical evidence of bleeding.

Guidelines on VTE prophylaxis should be clearly amended to encourage junior doctors to prescribe LMWH in the majority of CLD patients when there is an absence of absolute contraindications, and that the presence of CLD itself should not be perceived as a sufficient reason to withhold treatment. Implementation of such a strategy should be piloted, and would be likely to lead to a reduction in VTE events in CLD patients. By contrast, at present, there is evidence that many patients who would benefit from prophylactic LMWH are not receiving this important therapy.

\section{References}

1 Department of Health. Venous thromboembolism prevention: a patient safety priority. London: King's Thrombosis Centre, 2009.

2 Tripodi A, Mannucci PM. The coagulopathy of chronic liver disease. New Engl J Med 2011;365:147-56. 
3 Wu H, Nguyen G. Liver cirrhosis is associated with venous thromboembolism among hospitalized patients in a nationwide US study. Clin Gastroenterol Hepatol 2010;8:800-5.

4 Villa E, Cammà C, Marietta $\mathrm{M}$ et al. Enoxaparin prevents portal vein thrombosis and liver decompensation in patients with advanced cirrhosis. Gastroenterology 2012;143:1253-60.

5 Søgaard KK, Horváth-Puhó E, Grønbæk H et al. Risk of venous thromboembolism in patients with liver disease: a nationwide population-based case-control study. Am J Gastroenterol 2009;104:96-101

6 Buresi M, Hull R, Coffin CS. Venous thromboembolism in cirrhosis: A review of the literature. Can J Gastroenterol 2012;26:905-90.

7 National Institute for Health and Care Excellence. Venous thromboembolism: reducing the risk: Reducing the risk of venous thromboembolism (deep vein thrombosis and pulmonary embolism) in patients admitted to hospital. London: National Institute for Health and Care Excellence, 2010.

8 Dabbagh O, Oza A, Prakash S et al. Coagulopathy does not protect against venous thromboembolism in hospitalized patients with chronic liver disease. Chest 2010;137:1145-9.
9 Aldawood A, Arabi Y, Aljumah A et al. The incidence of venous thromboembolism and practice of deep venous thrombosis prophylaxis in hospitalized cirrhotic patients. Thromb J 2011;9:1.

10 Intagliata NM, Henry ZH, Shah N et al. Prophylactic anticoagulation for venous thromboembolism in hospitalized cirrhosis patients is not associated with high rates of gastrointestinal bleeding. Liver Int 2014;34:26-32.

11 Lloyd NS, Douketis JD, Cheng J at al. Barriers and potential solutions toward optimal prophylaxis against deep vein thrombosis for hospitalized medical patients: a survey of healthcare professionals. J Hosp Med 2012;7:28-34.

12 Stachow E, Berry M. An electronic prescribing system can ensure thromboprophylaxis is considered. Clin Med 2011;11:633.

13 Durieux P, Nizard R, Ravaud P et al. A clinical decision support system for prevention of venous thromboembolism: effect on physician behaviour. JAMA 2000;283:2816-21.

Address for correspondence: Dr D Sherman, Central Middlesex Hospital, Acton Lane, Park Royal, London NW10 7NS, UK.

Email: david.sherman@nhs.net 